Artículos

\title{
Ajuste escolar percibido por el profesor (PROF-A) en estudiantes mexicanos: análisis psicométrico
}

\author{
School Adjustment Perceived by the Teacher (PROF-A) in Mexican Students: Psychometric \\ Analysis
}

María Esthela Valenzuela-Santoyo ${ }^{1}$, Samuel Alejandro Portillo Peñuelas ${ }^{2}$, Jaime Fuentes-Balderrama ${ }^{3}$, Oscar Ulises Reynoso González ${ }^{4}$, Alba del Carmen Valenzuela-Santoyo ${ }^{1}$

1 Secretaría de Educación y Cultura (SEC-SONORA)

2 Instituto Tecnológico de Sonora

3 Universidad de Texas

4 Centro Universitario de Los Altos de la Universidad de Guadalajara

\section{Resumen}

El presente trabajo de investigación tuvo como objetivo validar la escala de ajuste escolar percibido por el profesor (PROF-A) de Cava et al. (2015) en estudiantes mexicanos de educación primaria. El diseño fue de tipo instrumental y el estudio se desarrolló con una muestra no probabilística de bola de nieve conformada por 37 profesores pertenecientes a ocho escuelas de una zona escolar de educación primaria en un municipio del sur del estado de Sonora, México; quienes evaluaron el ajuste escolar de los estudiantes adscritos a su grupo, obteniendo un total de 628 registros. Los análisis se llevaron a cabo en SPSS v.25 y R 4.0.1 utilizando los paquetes MVR, Lavaan, Psych y semPlot. Se realizó un análisis factorial confirmatorio y un análisis de consistencia interna para comprobar la validez y confiabilidad de la escala, obteniendo un modelo de cuatro factores (integración social, competencia académica, implicación familiar y relación profesor-alumno) al igual que en la escala original. Los resultados demuestran que la PROF-A puede ser utilizada en estudios posteriores con muestras mexicanas en el nivel de educación primaria, ya que muestra propiedades psicométricas satisfactorias; se recomienda su uso en el contexto mexicano.

Palabras clave: ajuste escolar percibido, análisis factorial confirmatorio, educación primaria, estudiantes, percepción del profesor.

María Esthela Valenzuela-Santoyo, Secretaría de Educación y Cultura (SEC-SONORA)

https://orcid.org/0000-0003-0470-4817

Samuel Alejandro Portillo Peñuelas, Departamento de Educación, Instituto Tecnológico de Sonora

http://orcid.org/0000-0002-1521-6619

Jaime Fuentes-Balderrama, Escuela de Trabajo Social Steve Hicks de la Universidad de Texas

http://orcid.org/0000-0002-8225-0294

Oscar Ulises Reynoso González, Centro Universitario de Los Altos de la Universidad de Guadalajara

http://orcid.org/0000-0002-0598-4665

Alba del Carmen Valenzuela-Santoyo, Secretaría de Educación y Cultura (SEC-SONORA)

https://orcid.org/0000-0002-9433-8779

Autor para correspondencia: samuel.portillo40519@potros.itson.edu.mx 


\begin{abstract}
The purpose of this research was to validate the Teacher Perceived School Adjustment Scale (PROF-A) developed by Cava et al. (2015) in Mexican elementary school students. Following an instrumental research design, the study was developed with a non-probabilistic snowball sample made up of 37 teachers belonging to eight schools in an elementary school zone from a municipality in the southern part of the state of Sonora, Mexico, who evaluated the school adjustment of the students in their class, obtaining a total of 628 records. The analyses were carried out in SPSS v.25 and R 4.0.1 using the MVR, Lavaan, Psych and semPlot packages. A confirmatory factor analysis and an internal consistency analysis were performed to test the validity and reliability of the scale which obtained a four-factor model (social integration, academic competence, family involvement and teacher-student relationship) as in the original scale. The results demonstrate that the PROF-A can be used in subsequent studies with Mexican samples at elementary school level as it displays satisfactory psychometric properties, and its use in the Mexican context is recommended.
\end{abstract}

Keywords: perceived school adjustment, confirmatory factor analysis, elementary school education, students, teacher's perception.

DOI https://doi.org/10.36793/psicumex.v11i2.414

Recibido: 05 - febrero - 2021

Aceptado 24 - Mayo - 2021

Publicado 25 - Septiembre - 2021 


\section{Introducción}

La escuela es por excelencia el lugar que se ha privilegiado para el desarrollo formal del aprendizaje, escenario al que en la actualidad se le ha sumado la participación de la familia en el desarrollo psicosocial del estudiante (Musitu et al., 2006). En este sentido, se reconoce que tanto en el espacio escolar como en el familiar se van adquiriendo y desarrollando conductas y actitudes que intervienen en los procesos de aprendizaje de los alumnos de manera complementaria. Si a ello se agregan las condiciones contextuales, se pueden identificar con mayor precisión aspectos que potencian o limitan los procesos cognitivos del estudiante.

Desde la perspectiva anterior, el indicador académico basado en las notas escolares dejaría de considerarse como del todo determinante para la comprensión del estudiantado. Por lo que se debe virar la mirada hacia los factores contextuales y personales que inciden en su actuar en el centro escolar para tener una visión global del sujeto. Dicha situación se refiere al ajuste escolar, el cual de manera general se ha concebido como el nivel de adaptación que muestra el estudiante ante las demandas de la escuela y su compromiso hacia las actividades escolares (Droguett, 2011), vinculado a la satisfacción y al aprovechamiento del ambiente escolar en el que se desarrolla (Bernal y Gálvez, 2017).

Si bien, no se ha consolidado una sola definición, se han ido integrando elementos para su comprensión. A saber, Mera et al. (2014) consideran relevante el grado de satisfacción que muestran los estudiantes durante las actividades escolares, mientras que otros autores añaden aspectos asociados al rendimiento académico, a la adaptación a las normas escolares, al respeto al profesor como figura de autoridad, a la actitud del estudiante hacia la escuela, a la participación en actividades escolares y a la integración del adolescente entre sus iguales con el fin de consolidar una mirada más integrativa del ajuste escolar (Heras y Navarro, 2012). 
Por su parte, Fernández-Lasarte et al. (2018), desde el punto de vista psicoeducativo, visualiza el ajuste escolar como un factor prioritario para la identificación y comprensión de problemas asociados a fracaso escolar, ausentismo y convivencia al momento de profundizar en el análisis de la dinámica escolar en términos de desajuste. De esta manera, dota de importancia tanto a la parte psicológica como educativa en la que se desenvuelve el estudiante.

Empíricamente, el ajuste escolar se ha relacionado con el autoconcepto (Cava et al., 2015; Droguett, 2011; Estévez et al., 2007; Fernández-Lasarte et al., 2018), el clima escolar y el apoyo social (MartínezFerrer et al., 2012; Pérez et al., 2020), la inteligencia emocional (Calero et al., 2018; Mera et al., 2014), el rendimiento académico (Antonio-Agirre et al., 2015; Bernal y Gálvez, 2017; Cava et al., 2015; Díaz y Ruiz, 2018; Fernández-Lasarte et al., 2019; Moral et al., 2010), la satisfacción con la vida (Azpiazu et al., 2014; Droguett, 2011; Martínez-Antón et al., 2007; Martínez-Ferrer et al., 2012) y con variables asociadas a contextos familiares y de violencia escolar (Cava et al., 2010; Estévez y Jiménez, 2014; Heras y Navarro, 2012; Martínez-Ferrer et al., 2012; Musitu et al., 2006; Valdez et al., 2012; Vera et al., 2014), así como con al bienestar subjetivo (Gutiérrez y Goncalves, 2013).

Referente a los procesos de recogida de información, el ajuste escolar se ha trabajado con escalas de autoinforme, desde la propia perspectiva del estudiante, siendo la escala breve de ajuste escolar (EBAE-10) de Moral et al. (2010) la más utilizada para analizar dicho constructo (Bernal y Gálvez, 2017; FernándezLasarte et al., 2019; Heras y Navarro, 2012; Pérez et al., 2020; Rodríguez-Fernández et al., 2018). Otros autores han utilizado las notas escolares como indicadores de rendimiento escolar, asociando mayores calificaciones a mayor ajuste en la escuela a nivel académico (Droguett, 2011; Valdez et al., 2012), mientras que otros han utilizado registros de notas negativas y reportes de los estudiantes en los centros escolares para búsqueda de desajuste en la escuela (Díaz y Ruiz, 2018; Estévez y Jiménez, 2014; Valdez et al., 2012). En 
todos los casos, es el estudiante quien dota dicha información de manera expresa, o bien, se toman como referencia los registros de desempeño académico y conductual.

En menor medida, se han desarrollado investigaciones en las que el ajuste escolar es producto de la percepción del profesorado sobre sus estudiantes (Cava et al., 2010; Cava et al., 2015; Estévez et al., 2007; Jiménez et al., 2008; Martínez-Antón et al., 2007; Martínez-Ferrer et al., 2012). Es esta última perspectiva la de interés para la presente investigación, ya que profundiza la mirada en el estudiantado en función de su desempeño y niveles de logro a nivel personal, académico y social. Inclusive, alude al nivel de participación por parte de la familia del estudiante.

Para efectos de la presente investigación, el ajuste escolar a nivel general se concibió como un aspecto que en términos positivos caracteriza al buen estudiante, al grado de motivación que presenta por asistir a la escuela y realizar las tareas escolares, a la habilidad para hacer amigos y relacionarse con los superiores, así como al sentirse parte del centro escolar. En contraparte, el estudiante que presenta escasa motivación por la escuela y las tareas escolares, conductas antisociales, disruptivas y violentas, así como desafiantes ante la autoridad escolar, además de insatisfacción y sentirse ajeno a sus pares exhibe lo que denominamos como desajuste escolar.

Por otra parte, en lo que respecta al ajuste escolar percibido por el profesor, en este estudio se considera la valoración que el profesional de la educación realiza de cada uno de sus estudiantes respecto a factores académicos, sociales y familiares que intervienen en el aprendizaje (motivación e interés por asistir a la escuela), convivencia (relación con compañeros), apoyo recibido (social y familiar) y en los niveles de logro (rendimiento académico) para su desarrollo en el centro escolar.

En relación con lo anterior, el objetivo fue validar la escala ajuste escolar percibido por el profesor (PROF-A) en una muestra de profesores que evaluaron estudiantes mexicanos de educación primaria. A saber, representa el primer estudio que se realiza con dicho instrumento en el país por lo que se proyecta 
como un aporte a la investigación educativa y psicológica en México. Se partió de la certeza de que el instrumento contaba con evidencias de validez y confiabilidad en una muestra española, y por lo tanto, podía ser utilizado en investigaciones realizadas con estudiantes mexicanos de educación primaria para medir el ajuste escolar.

\section{Metodología}

De acuerdo con el modelo de Ato et al. (2013), el diseño es de tipo instrumental, ya que su objetivo central fue la revisión de las propiedades psicométricas de la escala PROF-A.

\section{Participantes}

Para conformar la muestra del estudio, se acudió a una supervisión escolar de un municipio del sur de Sonora, México. A través de una reunión con directores, se solicitó su apoyo para contactar a los docentes de sus respectivas escuelas para responder el instrumento. En dicho sentido, mediante un muestreo no probabilístico de bola de nieve, se conformó un grupo de 37 docentes de ocho instituciones distintas de educación primaria. Particularmente, el $31 \%$ eran hombres y el $69 \%$ mujeres, con una edad promedio de 34 años $(D E=8.30)$. El profesorado pertenece a una zona escolar ubicada en el contexto urbano y cuentan con al menos 5 años

de experiencia docente. A su vez, cada uno de ellos respondió la prueba de PROF-A para cada uno de los estudiantes adscritos a su grado, y se obtuvo una muestra total de 628 registros. Específicamente, el $56.4 \%$ de los estudiantes eran niñas y el $43.6 \%$ niños, con edades entre los 6 y los 11 años $(M=8.57, D E=1.66)$. En cuestión del grado de estudio, la distribución fue equilibrada, ya que el $14.2 \%$ era de primero, el $14.6 \%$ de segundo, el 16. $9 \%$ de tercero, el $18.9 \%$ de cuarto, el $18.2 \%$ de quinto y el $17.2 \%$ de sexto año de primaria.

\section{Instrumento}

Escala de ajuste escolar percibido por el profesor (Cava et al., 2015). Ésta es una versión extendida y revisada del instrumento de Cava y Musitu (1999), el cual fue diseñado con base en la propuesta de García-Bacete 
(1989). La escala está constituida por 14 reactivos y cuatro factores relacionados: (1) integración social (ítems 1, 2, 3 y 4), que alude a la relación del estudiante con sus pares dentro del aula (p. ej. "la facilidad del alumno/a en formar parte de grupos de amigos/as en clase”); (2) competencia académica (ítems 5, 6, 7 y 8), que aborda el compromiso e involucramiento del estudiante con las tareas y actividades (p ej. "la participación del alumno/a en las actividades, discusiones, debates, que se proponen en clase"); (3) implicación familiar (ítems 9, 10, 11 y 12), que identifica la participación y soporte del núcleo familiar (p. ej. “grado de implicación de la familia en el seguimiento escolar del hijo/a.”) y (4) Relación profesor alumno (ítems 13 y 14), que detalla el grado en que el docente se interesa por cada uno de sus estudiantes (p. ej. “el tiempo que dedico a hablar con este alumno/a"). Todos los reactivos son respondidos por el docente para cada uno de los estudiantes de su grupo que se pretenden evaluar. Cuenta con un formato de respuesta que va de 1 (muy bajo) a 10 (muy alto). Los autores reportan propiedades psicométricas satisfactorias, tanto de confiabilidad constatada a través de la revisión de la consistencia interna (integración social .95, implicación familiar .91, competencia académica .92 y relación profesor-alumno de .88. Valor total de la escala .91) como de validez $\left(\mathrm{SB} X^{2}=345.37, \mathrm{gl}=62, \mathrm{p}<.001, \mathrm{CFI}=.972, \mathrm{NNFI}=.960, \mathrm{NFI}=.967, \mathrm{RMSEA}=.065\right)$ en las respuestas dadas sobre una muestra de adolescentes españoles.

\section{Procedimiento}

Después de solicitar la autorización de los autores, se realizó una revisión general del instrumento, en donde se determinó mantener todos los reactivos en su forma original. Después de conformar la muestra del estudio, la aplicación de la escala a la muestra seleccionada se realizó mediante el envío y recepción de datos vía correo electrónico. A cada docente se le hizo llegar una plantilla para la valoración del ajuste escolar de cada uno de los estudiantes adscritos a su grupo. Llenado el formato, se enviaron los datos al equipo de investigación para la conformación de la base de datos. 


\section{Análisis de datos}

Una vez capturada y depurada la información, se efectuó un análisis factorial confirmatorio y un análisis de consistencia interna. Los análisis se llevaron a cabo en $S P S S$ v.25 y en $R$ 4.0.1 utilizando los paquetes $M V R$, Lavaan, Psych y semPlot.

\section{Consideraciones éticas}

La participación de los docentes fue voluntaria y con consentimiento informado de uso exclusivo de datos para análisis estadísticos y presentación de informes con fines académicos, garantizando los principios de participación anónima, sin alguna compensación a cambio

\section{Resultados}

\section{Análisis factorial confirmatorio}

Al analizar las distribuciones univariadas, se aprecia que los 14 ítems del PROF-A siguieron una distribución aproximadamente normal pues ninguno presenta sesgo mayor a 2 ni curtosis mayor a 6 (Curran et al., 1996). Pese a que el conjunto de variables no sigue una distribución multivariada normal $\left(M_{\text {sesgo }}=2988, p<.01 y\right.$ $\left.M_{\text {curtosis }}=43.91, p<.01\right)$, no presenta curtosis multivariada negativa, lo cual permite el uso de un estimador robusto ante la no normalidad (i.e. máxima verosimilitud ajuste a media y varianza con errores estándar robustos), sin el riesgo de inflar el error tipo I.

El modelo se especificó siguiendo la estructura original. Pese a que el factor 4 relación profesor alumno se encuentra no identificado por sí mismo, el modelo total sí está identificado y permite la estimación de la varianza latente y el resto de los parámetros del cuarto factor al correlacionar sus errores con errores de otros indicadores exógenos (Kline, 2011). Para mejorar el ajuste del modelo base, se agregaron cuatro índices de modificación: correlación entre ítems 1 y 4 (relativos al ajuste social del alumno), 6 y 11 (nivel de esfuerzo del alumno y participación parental), 9 y 13 (implicación familiar e interés del alumno), así 
como 12 y 14 (comunicación familia con docentes y comunicación docente-alumno). La figura 1 muestra el diagrama obtenido.

\section{Figura 1}

Análisis factorial confirmatorio de la escala de ajuste escolar percibido por el profesor (PROF-A)

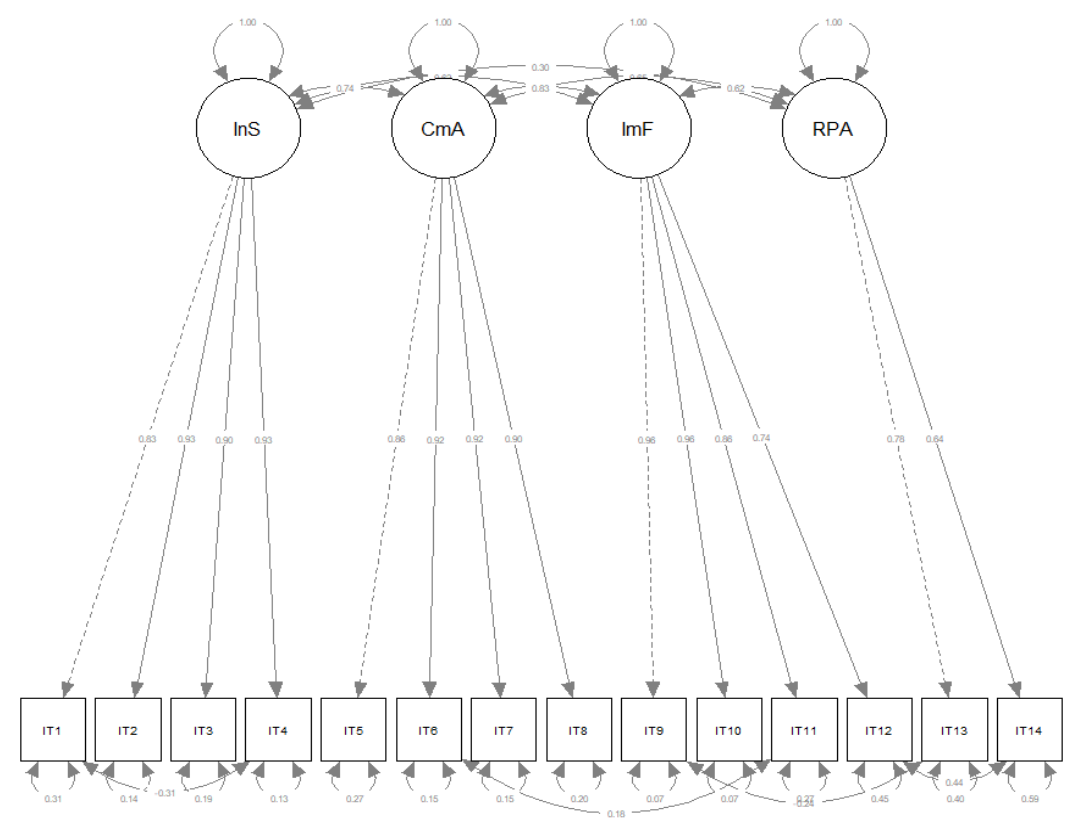

Fuente: Elaboración propia con base en el paquete semPlot.

Como se puede observar en la figura 1 , se obtienen cargas factoriales superiores a .6. Todas las cargas factoriales y varianzas latentes fueron significativas $\mathrm{y}$, adicionalmente, los ítems presentaron niveles moderados y altos de varianza explicada $\left(R^{2}: 0.4-0.93\right)$.

El ajuste absoluto del modelo fue evaluado mediante la chi cuadrada ajustada de Satorra-Bentler donde un valor significativo indica siquiera un punto de discrepancia entre la matriz de varianza-covarianza implicada y la matriz observada. Al no contar con un ajuste absoluto, se evalúa el ajuste cercano por medio del índice de ajuste comparativo (Comparative Fit Index [CFI]) y del índice de Tucker-Lewis (Tucker-Lewis 
Index $[\mathrm{TLI}])$, donde valores mayores a .9 indican un buen ajuste y superiores a .95 indican un excelente ajuste. Con respecto a los residuales, se evaluaron la raíz cuadrada del promedio de los residuales (Root Mean Square Residual [SRMR]) y el error de aproximación cuadrático medio (Root Mean Square Error of Approximation [RMSEA]) donde valores debajo de .08 indican un buen ajuste y debajo de .05 un muy buen ajuste. La tabla 1 muestra los índices de ajuste obtenidos en el análisis factorial confirmatorio.

\section{Tabla 1}

Índices de ajuste

\begin{tabular}{lllll}
\hline $\boldsymbol{X}^{2}$ (67) & CFI & TLI & RMSEA & SRMR \\
\hline $264.85^{* * *}$ & .97 & .96 & $.07^{* *}[$ IC90\% .06-.07] & .04 \\
\hline Fuente: Elaboración propia con base en los resultados de Lavaan. $* p<.05, * * p<.01, * * * p<.001$.
\end{tabular}

Como se presenta en la tabla 1 , los indicadores demuestran un muy buen ajuste al modelo, ya que los valores reportados cumplen con los criterios de adecuación (Byrne, 2016). En dicho sentido, se confirma que la PROF-A presenta validez de constructo en una muestra mexicana.

\section{Confiabilidad}

Finalmente, se reportó la consistencia interna de cada dimensión a través del coeficiente alfa de Cronbach. En los tres primeros factores se obtuvieron valores satisfactorios $(\alpha>.94-.95)$. Sin embargo, no fue el caso para la dimensión relación profesor alumno, la cual obtuvo un valor bajo $(\alpha=.69)$ (Abad et al., 2011). Esta situación podría deberse a la poca cantidad de reactivos en la dimensión y coincide con la escala original en ser el factor que presenta el coeficiente de consistencia interna más bajo de la escala (Cava et al., 2015). La PROF-A obtuvo una alta consistencia interna total $(\alpha=.94)$. Las consistencias internas de cada subescala, correlaciones de cada ítem con los demás en su subescala, así como la correlación de los ítems con todos los que conforman el instrumento en su totalidad se pueden observar en la tabla 2. 
Tabla 2

Consistencia interna de subescalas y correlaciones inter-ítem

\begin{tabular}{|c|c|c|}
\hline Ítem & $\begin{array}{l}\text { Correlación } \\
\text { subescala }\end{array}$ & $\begin{array}{l}\text { Correlación } \\
\text { total }\end{array}$ \\
\hline \multicolumn{3}{|l|}{ Integración social $(\alpha=.94)$} \\
\hline 1. El grado de ajuste social del alumno/a en el aula. & 0.79 & 0.64 \\
\hline 2. La relación del alumno/a con sus compañeros/as. & 0.90 & 0.71 \\
\hline $\begin{array}{l}\text { 3. El interés del alumno/a en conocer y hacer amistad con sus } \\
\text { compañeros. }\end{array}$ & 0.87 & 0.73 \\
\hline $\begin{array}{l}\text { 4. La facilidad del alumno/a en formar parte de grupos de amigos/as } \\
\text { en clase. }\end{array}$ & 0.86 & 0.74 \\
\hline \multicolumn{3}{|l|}{ Competencia académica $(\alpha=.94)$} \\
\hline 5. Media aproximada del rendimiento académico actual del alumno/a. & 0.81 & 0.83 \\
\hline 6. Nivel de esfuerzo del alumno (no considerar rendimiento). & 0.89 & 0.84 \\
\hline 7. El interés y la atención del alumno en lo que se hace en clase. & 0.89 & 0.83 \\
\hline $\begin{array}{l}\text { 8. La participación del alumno/a en las actividades, discusiones, } \\
\text { debates que se proponen en clase. }\end{array}$ & 0.86 & 0.84 \\
\hline \multicolumn{3}{|l|}{ Implicación familiar ( $\alpha=.93$ ) } \\
\hline $\begin{array}{l}\text { 9. Grado de implicación de la familia en las tareas escolares del } \\
\text { hijo/a. }\end{array}$ & 0.91 & 0.85 \\
\hline $\begin{array}{l}\text { 10. Grado de implicación de la familia en el seguimiento escolar del } \\
\text { hijo/a. }\end{array}$ & 0.92 & 0.85 \\
\hline 11. Grado de participación de los padres en el centro escolar. & 0.81 & 0.77 \\
\hline $\begin{array}{l}\text { 12. Grado en que la comunicación entre padres y profesores es } \\
\text { abierta y fluida. }\end{array}$ & 0.72 & 0.69 \\
\hline \multicolumn{3}{|l|}{ Relación profesor-alumno ( $\alpha=.66)$} \\
\hline 13. El interés personal que muestro a este alumno/a. & 0.49 & 0.47 \\
\hline 14. El tiempo que dedico a hablar con este alumno/a. & 0.49 & 0.45 \\
\hline
\end{tabular}

Fuente: Elaboración propia con base en los resultados del paquete Psych. 


\section{Discusión}

Se confirma que la PROF-A muestra propiedades psicométricas satisfactorias, pues presenta validez de constructo (análisis factorial confirmatorio) y confiabilidad alta (análisis de consistencia interna) en la percepción del profesorado hacia el ajuste escolar del estudiantado en educación primaria (niños mexicanos entre 6 y 12 años). En este sentido, se permiten identificar cuatro factores: integración social (relativo a la percepción del profesor sobre la dinámica social del estudiante en el aula), implicación familiar (relacionado a la participación de los padres de familia en el centro escolar), competencia académica (alude al nivel de desempeño mostrado por el alumno en el aula) y relación profesor-alumno (relacionado al tiempo e interés que mantiene el profesor por el alumno).

Si bien, la literatura se ha centrado en estudiar el ajuste escolar en educación secundaria con muestras adolescentes (Antonio-Agirre et al., 2015; Bernal y Gálvez, 2017; Fernández-Lasarte et al., 2018; Heras y Navarro, 2012), así como en educación media con jóvenes (Azpiazu et al., 2014; Díaz y Ruiz, 2018; Valdez et al., 2012), el presente estudio muestra que también puede ser estudiado desde el nivel de educación primaria a través de la aplicación de la PROF-A. Sin embargo, no es el alumnado quien responde directamente, sino el profesorado quien con base en la observación del estudiantado dentro y fuera del aula evalúa el grado de integración, el nivel de participación, el desempeño y el tiempo de atención que requiere en la escuela.

En este sentido, se coincide con Cava et al., (2015) en cuanto a que el uso de la PROF-A permite al profesorado reflexionar y prestar atención a los estudiantes adscritos a su grupo. Dicho ejercicio permite identificar por alumno situaciones que requieren apoyo, estas relacionadas a la convivencia, al apoyo social y familiar, a la competencia y habilidades académicas, a las relaciones que mantienen entre iguales (alumnoalumno), y entre el profesor y el alumno. A saber, Cava et al. (2010) señala cómo el ajuste escolar percibido por el profesor ha sido relacionado con violencia escolar, mientras que Estévez et al. (2007) ha documentado 
su relación con las conductas agresivas del estudiantado. En este sentido, la mirada del profesor hacia sus estudiantes permite no sólo observar el desempeño hacia cuestiones de carácter académico, sino también asociado a su autoconcepto (Cava et al., 2015) el estatus social del estudiante en el aula (Jiménez et al., 2008), el clima escolar y la satisfacción con la vida (Martínez-Antón et al., 2007; Martínez-Ferrer et al., 2012).

\section{Conclusión}

A partir de la aplicación de la PROF-A en una muestra de estudiantes mexicanos, se concluye que la escala puede ser utilizada en estudios posteriores en el nivel de educación primaria debido a los indicadores aceptables de validez de constructo y confiabilidad que presenta. Además, se destaca la relevancia de la aplicación de dicha escala para propiciar el reconocimiento de los estudiantes adscritos al grupo que es atendido por el profesorado, así como de orientar la mirada hacia la integración social, la competencia académica, la implicación familiar y la relación profesor-estudiante como los principales factores para explicar el ajuste escolar.

Con base en la información que el profesorado obtenga de cada uno de sus estudiantes, tanto a nivel individual como grupal, podrá optar por tomar mejores decisiones dentro y fuera del aula que favorezcan la formación integral del estudiantado para la mejora de su proceso de formación. En este sentido, se sugiere replicar el estudio aplicando la escala en otros contextos (sectores urbanos marginados y/o rurales) y escenarios educativos (p. ej. escuelas con población migrante e indígena, escuelas de organización multigrado, escuelas con jornadas de tiempo completo) e inclusive determinar el grado de concordancia entre la evaluación docente y la autoreportada por los estudiantes. Asimismo, valdría la pena conocer en estudios posteriores cómo se vincula la PROF-A con el rendimiento académico y otras variables de interés.

En cuanto a las limitaciones del estudio, es necesario hacer mención de algunos aspectos. Primero, si bien se obtuvo una cantidad adecuada de observaciones para efectuar los análisis, éstas provienen 
únicamente de 37 docentes. Además, ya que cada uno de ellos debe responder por la totalidad de los estudiantes, la instrumentación podría presentarse como una amenaza a la validez (Salkind, 1999). Segundo, es preciso advertir que la implementación correcta de la escala requiere que el docente tenga información suficiente y clara sobre cada uno de los estudiantes a evaluar, ya que algunos de los reactivos abordan no sólo el ajuste dentro del aula, sino también la implicación del núcleo familiar en sus actividades. Aunque esta condición se dio por sentada al considerar que el levantamiento de información se efectuó después de seis meses de haber iniciado el ciclo escolar, es pertinente confirmar que el docente es capaz de identificar tales elementos. En dicho sentido, se sugiere que en próximos estudios, previo a su aplicación, se considere el tiempo que el respondiente ha pasado con los estudiantes y se determine si es idóneo para la aplicación de la escala.

\section{Conflicto de intereses}

Los autores declaran no tener conflicto de intereses

\section{Financiamiento}

Ninguno

\section{Agradecimientos}

Ninguno

\section{Referencias}

Abad, F., Olea, J., Ponsoda, V. y García, C. (2011). Medición en ciencias sociales y de salud. Madrid: Síntesis.

Antonio-Agirre, I., Azpiazu, L., Esnaola, I. y Sarasa, M. (2015). Capacidad predictiva del autoconcepto y la inteligencia emocional en el ajuste escolar autopercibido. Bordón. Revista de Pedagogía, 67(4), 925. https://doi.org/10.13042/Bordon.2015.67401 
Ato, M., López, J. y Benavente, A. (2013). Un sistema de clasificación de los diseños de investigación en psicología. Anales de psicología, 29(3), 1038-1059. https://doi.org/10.6018/analesps.29.3.178511 Azpiazu, L., Esnaola, I. y Ros, I. (2014). Factores contextuales y variables individuales en el ajuste escolar. Revista INFAD de Psicología. International Journal of Developmental and Educational Psychology, 6(1), 327-336. https://doi.org/10.17060/ijodaep.2014.n1.v6.751

Bernal, F. y Gálvez, F. (2017). Ajuste escolar y rendimiento académico y su relación con la motivación y el autoconcepto. Análisis de diferencias en muestra chilena y española. Revista De Orientación Educacional, 31(60), 2-21. http://www.roe.cl/index.php/roe/article/view/21

Byrne, B. (2016). Structural Equation Modeling with Amos. New York: Routledge.

Calero, A., Barreyro, J., Formoso, J. y Injoque, I. (2018). Inteligencia emocional y necesidad de pertenencia al grupo de pares durante la adolescencia. Subjetividad y Procesos Cognitivos, 22(2), 38-56.

Cava, M., Buelga, S., Musitu, G. y Murgui, S. (2010). Violencia escolar entre adolescentes y sus implicaciones en el ajuste psicosocial: un estudio longitudinal. Revista de Psicodidáctica, 15(1), 21 34. https://ojs.ehu.eus/index.php/psicodidactica/article/view/732

Cava, M., Povedano, A., Buelga, S. y Musitu, G. (2015). Análisis psicométrico de la escala de ajuste escolar percibido por el profesor (PROF-A). Psychosocial Intervention, 24, 63-69. https://doi.org/10.1016/j.psi.2015.04.001

Cava, M. y Musito, G. (1999). Percepción del profesor y estatus sociométrico en el grupo de iguales. Información Psicològica, 71, 60-65.

Curran, P., West, S. y Finch, J. (1996). The Robustness of Test Statistics to Nonnormality and Specification Error in Confirmatory Factor Analysis. Psychological Methods, 1(1), 16-29. https://doi.org/10.1037/1082-989X.1.1.16 
Díaz, D. y Ruiz, A. (2018). Reprobación escolar en el nivel medio superior y su relación con el autoconcepto en la adolescencia. Revista Latinoamericana de Estudios Educativos, 48(2), 125-142. https://doi.org/10.48102/rlee.2018.48.2.49

Droguett, L. (2011). Rasgos psicológicos asociados al ajuste social y personal de alumnado adolescente [Tesis doctoral]. Universidad del País Vasco.

Estévez, E., Murgui, S., Moreno, D. y Musitu, G. (2007). Estilos de comunicación familiar, actitud hacia la autoridad institucional y conducta violenta del adolescente en la escuela. Psicothema, 19, 108-113. http://www.psicothema.com/pdf/3335.pdf

Estévez, E. y Jiménez, T. (2014). Conducta agresiva y ajuste personal y escolar en una muestra de estudiantes adolescentes $\quad$ españoles. $\quad$ Universitas $\quad$ Psychologica, $111-124$. https://doi.org/10.11144/Javeriana.upsy14-1.caap

Fernández-Lasarte, O., Goñi, E., Camino, I. y Zubeldia, M. (2018). Ajuste escolar y autoconcepto académico en la Educación Secundaria. Revista De Investigación Educativa,37(1), 163-179. https://doi.org/10.6018/rie.37.1.308651

Fernández-Lasarte, O., Ramos-Díaz, E. y Axpe-Saez, I. (2019). Rendimiento académico, apoyo social percibido e inteligencia emocional en la universidad. European Journal of Investigation in Health, Psychology and Education (EJIHPE), 9(1), 39-49. https://doi.org/10.30552/ejihpe.v9i1.315

García-Bacete, F. (1989). Los niños con dificultades de aprendizaje y ajuste escolar: aplicación y evaluación de un modelo de intervención con padres y niños como coterapeutas [Tesis doctoral]. Universitat de València.

Gutiérrez, M. y Goncalves, T. (2013). Developmental Assets, School adjustment, and Adolescents' Subjective Well-being. International Journal of Psychology and Psychological Therapy, 13 (3), 339355. 
Heras, J. y Navarro, R. (2012). Ajuste escolar, soledad y conducta agresiva entre estudiantes de educación secundaria. Revista Qurrículum, 25, 105-124.

Jiménez, T., Moreno, D., Murgui, S. y Musitu, G. (2008). Factores psicosociales relacionados con el estatus social del alumno en el aula: El rol de la reputación social, la amistad, la conducta violenta y la relación con el profesor. International Journal of Psychology and Psychological Therapy, 8, 227 236.

Kline, R. (2011). Principles and Practice of Structural Equation Modeling. New York: Guilford Press.,

Martínez-Antón, M., Buelga, S. y Cava, M. (2007). La satisfacción con la vida en la adolescencia y su relación con la autoestima y el ajuste escolar. Anuario de Psicología, 38(2), 293-303. https://www.redalyc.org/articulo.oa?id=97017404013

Martínez-Ferrer, B., Povedano, A., Amador, L. y Moreno, D. (2012). Clima escolar, satisfacción con la vida y victimización en la escuela. Un análisis del efecto moderador del género. Anales de Psicología, 28(3), 875-882. http://dx.doi.org/10.6018/analesps.28.3.156121

Mera, M., Martínez-Taboad, C. y Elgorriaga, E. (2014). Rendimiento académico, ajuste escolar e inteligencia emocional en adolescentes inmigrantes y autóctonos. Boletín de Psicología, 1(110), 6982.

Moral, J., Sánchez, J. y Villareal, M. (2010). Desarrollo de una escala multidimensional breve de ajuste escolar. Revista Electrónica de Metodología Aplicada, 15(1), 1-11.

Musitu, G., Martínez, B. y Murgui, S. (2006). Conflicto marital, apoyo parental y ajuste escolar en adolescentes. Anuario de Psicología, 37(3) 247-258. https://revistes.ub.edu/index.php/Anuariopsicologia/article/view/8459/10442

Pérez, I., Zamora, M., Caldera, J., Reynoso, O., Cadena, A. y Mora, O. (2020). Ajuste escolar, clima escolar y apoyo social en bachilleres. Revista de Psicología y Ciencias del Comportamiento de la Unidad 
Académica de Ciencias Jurídicas y Sociales, 11(1), 5-18. https://doi.org/10.29059/rpcc.20200617100

Rodríguez-Fernández, A., Ramos-Díaz, E. y Axpe-Saez, I. (2018). The Role of Resilience and Psychological Well-Being in School Engagement and Perceived Academic Performance: An Exploratory Model to Improve Academic Achievement. In B. Bernal-Morales (Ed) Health and Academic Achievement (pp. 159-176). http://dx.doi.org/10.5772/intechopen.73580

Salkind, N. (1999). Métodos de investigación. México: Pearson Prentice Hall

Valdez, A., Sánchez, P. y Carlos, E. (2012). Autoconcepto social y ajuste escolar de estudiantes de educación media con conductas de hostigamiento en la escuela. Educación y ciencia, 2 (40), 85-96.

Vera, A., Ávila, M., Martínez, B. y Musitu, G. (2014). Relación entre familia y escuela y sus implicaciones en el ajuste escolar en adolescentes. En A. Bazán y N. Vega (Coords.), Familia-Escuela-Comunidad Teorías en la práctica (pp. 129-153). Ediciones mínimas.

Cómo citar este artículo: Valenzuela-Santoyo, M. E., Portillo Peñuelas, S. A., Fuentes-Balderrama, J., Reynoso González, O. U., \& ValenzuelaSantoyo, A. del C. (2021). Ajuste escolar percibido por el profesor (PROF-A) en estudiantes mexicanos: análisis psicométrico. Psicumex, 11, 118, e414. https://doi.org/10.36793/psicumex.v11i2.414 